\title{
Evaluación de la cadena de valor del cacao (Theobroma cacao I.) peruano
}

\section{(Assessment of the value chain of Peruvian cacao [Theobroma cacao I.])}

\author{
Guzmán-Bautista, $\mathrm{JH},{ }^{1}$ Chire-Fajardo, $G C^{2}$
}

\begin{abstract}
Resumen
La investigación tuvo como objetivo principal determinar las deficiencias de la situación actual de la cadena de valor del cacao peruano, y plantear propuestas de mejora integrales en la misma, a través de los factores competitivos en cuestión. Para ello se utilizaron como herramientas: el análisis de la cadena de valor y el análisis del diamante competitivo de Porter. El análisis de la cadena de valor, permitió una "fotografía" completa de quienes han participado, de qué manera, con qué intereses, sus interrelaciones, visualizando las necesidades y potencialidades de los diferentes actores para el mejoramiento individual y en conjunto. En el análisis del diamante competitivo de Porter se interrelacionaron los componentes del sector del cacao. Las mejoras en la cadena de valor fueron: el resultado de la vinculación de la estrategia, la estructura y la rivalidad entre las compañías del sector, la condición de los factores, las condiciones de la demanda, y los sectores conexos y de apoyo o clúster, que son actividades ligadas entre las empresas que participan en un sector del cacao; todas fundamentales para alcanzar la ventaja competitiva; que van a reducir la brecha de los diferentes eslabones convertidos en factores competitivos, para dar lugar al desarrollo de capacidades y generar oferta exportable de cacao en diferentes nichos de mercado, que a la fecha está alrededor de 100000 toneladas aproximadamente. Las operaciones de producción agrícola, pos cosecha y transformación requieren mejoras sustanciales para evitar costos de no calidad y la baja retribución del valor del cacao hacia el agricultor. De los problemas encontrados se derivan un conjunto de lineamientos estratégicos en el corto, mediano y largo plazo, proyectando para el futuro una cadena competitiva, como el posicionamiento por "diferenciación", direccionada a producir cacao fino o de aroma, cacao orgánico e innovación en productos con cacao peruano.
\end{abstract}

\section{Palabras clave}

Cacao orgánico; cadena de valor del cacao; clúster; estrategia de posicionamiento; factor competitivo, Perú.

\begin{abstract}
The main objective of the research was to determine the deficiencies of the current situation of the Peruvian cocoa value chain and pose an integral improvement in its proposals through the competitive factors in question. In order to achieve it, these tools were used: the analysis of the value chain and the analysis of Porter's competitive diamond. The analysis of the value chain allowed a complete 'photograph' of those who have participated, how, with what interests and their interrelations, allowing to visualize the needs and potential of the different actors for individual and joint improvement. In the analysis of Porter's competitive diamond, the components of the cocoa sector were interrelated. The improvements in the value chain were: the result of the linkage of the strategy, the structure and the rivalry between the companies of the sector, the condition of the factors, the conditions of the demand, and the related and support sectors or cluster, which are activities linked between the companies involved in a cocoa sector; all of them fundamental to achieve competitive advantage; that will reduce the gap of the different links turned into competitive factors, to give rise to the development of capacities and to generate exportable supply of cocoa in different niche markets, which, to date, is around 100000 tons. The operations of agricultural production, post-harvest and transformation requires substantial improvements to avoid non-quality costs and the low retribution of the value of cocoa to the farmer. From the problems encountered, a set of strategic guidelines is derived in the short, medium and long term, projecting a competitive chain for the future, such as positioning by 'differentiation', aimed at producing fine cocoa or aroma cocoa, organic cocoa and innovation in products with Peruvian cocoa.
\end{abstract}

\section{Keywords}

Cocoa value chain; competitive factor; cluster; organic cocoa; Peru; positioning strategy.

$1 \quad$ Universidad de Barranca, Barranca-Lima, Perú (jguzman@unab.edu.pe).

2 Universidad Nacional Agraria La Molina, Lima, Perú (gchire@lamolina.edu.pe) 


\section{Introducción}

La visita técnica de los autores a las zonas productoras de cacao del Perú, la revisión de publicaciones físicas y digitales, así como la recopilación de materiales desarrollados, fruto de sus experiencias en trabajos vinculados a la capacitación en fortalezas y habilidades de recursos humanos en comunidades y cooperativas agrarias cacaoteras de la Costa y elva, al igual que los cursos de extensión universitaria y proyección social realizados en las diferentes regiones del Perú y el apoyo de instituciones vinculadas a la cadena de valor; constituyen un esfuerzo invalorable y justificable para plantear problemas y poder delinear alternativas de solución, enmarcando aspectos de competitividad en el área agronómica, ambiental, de transformación y comercialización a nivel nacional, con propuestas de mejora tecnológica, acercándolo socialmente en forma transversal en las regiones cacaoteras del Perú.

Los objetivos del estudio fueron: 1. Determinar las deficiencias de la situación actual de la cadena de valor del cacao peruano, y plantear propuestas de mejora integrales en la misma, a través de los factores competitivos en cuestión; 2) Determinar los actores directos e indirectos de la cadena de valor del cacao.

\section{Metodología}

Zona de estudio. Son las regiones cacaoteras de San Martín, Junín, Cusco, Ucayali, Huánuco, Amazonas, Cajamarca y Piura, tomando como data valores de volúmenes de producción desde el año 2000 hasta el 2017.

Fuentes de información. La información, procesamiento e interpretación de datos de las regiones citadas, fue obtenida mediante entrevistas personales a los diferentes actores de la cadena de valor. Por otro lado, se utilizó la técnica de la observación, en la cual se tomó apuntes de las acciones y hechos que fueron de interés a lo largo de la cadena de valor. De las fuentes secundarias se obtuvo información de investigaciones similares recientes en la zona, halladas por trabajos de titulación, libros y revistas especializadas, publicaciones de conferencias nacionales e internacionales. La información también se obtuvo de anuarios estadísticos de instituciones gubernamentales como Ministerio de Agricultura y Riego (MINAGRI), Superintendencia Nacional de Aduanas y de Administración Tributaria (SUNAT), Instituto Nacional de Estadística e Informática (INEI) y organismos no gubernamentales (ONG), resaltando los volúmenes de producción, comercialización y exportación del cacao; rendimientos, usos de tecnología y aspectos socioeconómicos.

Tipo de Investigación. Es exploratoria y descriptiva, presentando aspectos transversales con la característica más usual que es la de recolección de datos. Asimismo, muestra aspectos longitudinales o evolutivos cuyos propósitos son de análisis de cambios a través del tiempo (Hernández, 2006). La producción de cacao en grano, por regiones, se ha conseguido de la pagina web del INEI, seleccionando "sistema de información regional para la toma de decisiones", luego "todos los departamentos del Perú", posteriormente como indicador "producción de cacao", seguidamente "anual, del año 2000 al 2016". Los precios del cacao se han conseguido de la página web de la Organización Internacional del Cacao (ICCO), ingresando a "statistics", luego a "cocoa prices", y seleccionando "monthly averages". 
Fase 1: Recopilación de Información.- Se recopiló y evaluó la información de las instituciones mencionadas que elaboran estadísticas nacionales e internacionales de la producción, de la exportación del cacao, así como de las fuentes primarias.

Fase 2: Aplicación de los modelos.- El estudio se realizó con los modelos planteados por Porter: el análisis de la cadena valor y el análisis del diamante competitivo de Porter.

El análisis de la cadena de valor: con este método se identificaron las fuentes de ventaja competitiva en las actividades que generan valor, descomponiendo sistemáticamente la cadena del cacao en cada una de las diferentes actividades. Mediante esta herramienta se determinó el valor del cacao, como la suma de los beneficios percibidos menos los costos percibidos al venderlo.

El análisis del diamante competitivo de Porter: es un enfoque que interrelaciona cuatro factores influenciados de una manera proactiva (Figura 1):

1. La estrategia, la estructura y la rivalidad entre las compañías que contribuyen a crear reglas de juego claras y estables.

2. La condición de los factores describe elementos de ventaja comparativa; mano de obra, tierra y capital, además de las condiciones agrológicas, climáticas y geográficas.

3. Las condiciones de la demanda por parte de consumidores nacionales e internacionales.

4. Los sectores conexos y de apoyo o clúster (Porter, 1990).

Figura 1. Metodología utilizando el diamante de Porter

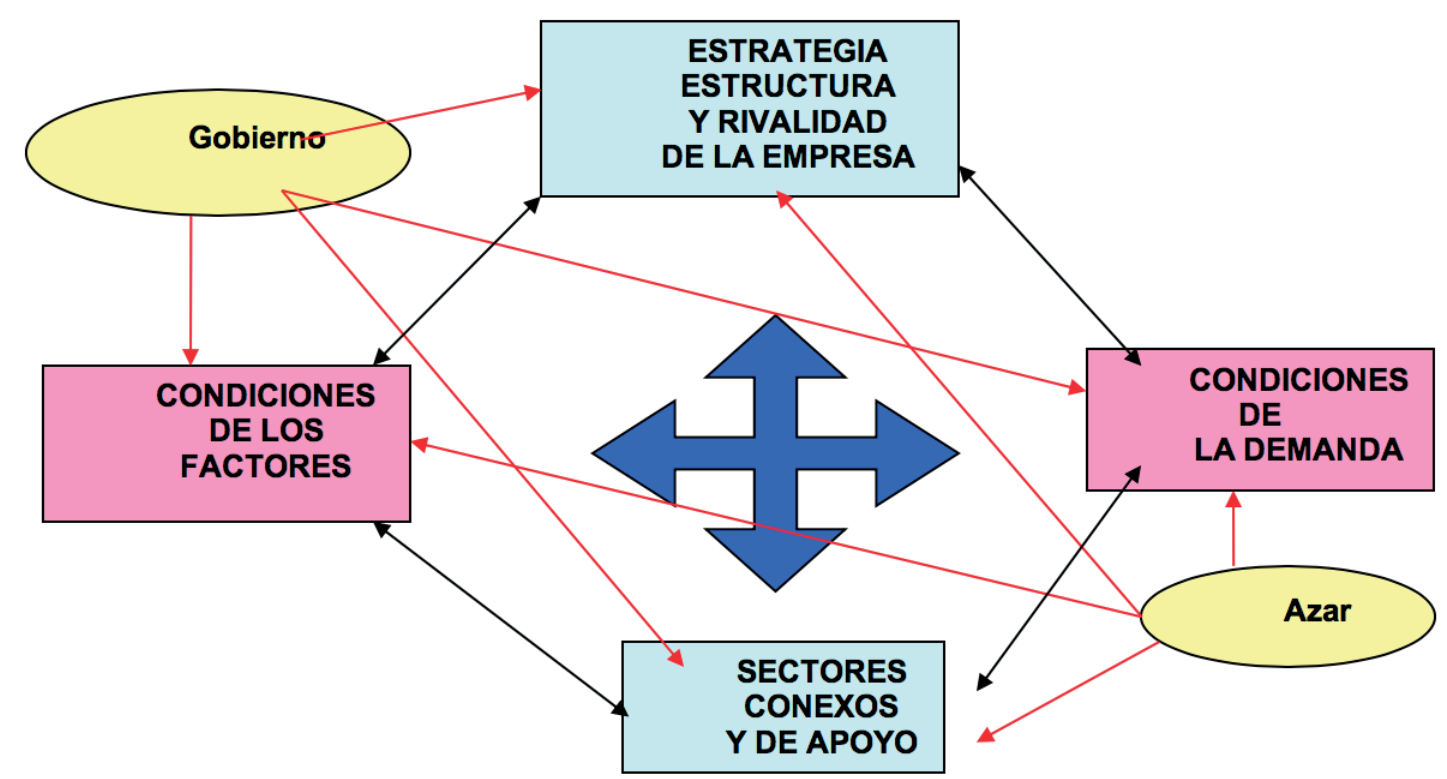

\section{Resultados y discusión}

En el análisis de la cadena de valor, se segmenta dicha cadena por actividades, con la finalidad de diagnosticar las deficiencias; por ejemplo, el productor sabe cómo produce y cómo entrega el cacao al acopiador, pero no siempre sabe cómo el grano de cacao se procesa, se comercializa y se exporta; desconoce cómo se realiza la selección, cuánto descarte se genera y por qué el precio de ese grano es mucho mayor al que le pagan en campo, por lo tanto, este segmento 
de la cadena se denomina "El productor". Por otra parte, "El acopiador", de igual manera, desconoce las etapas posteriores de procesamiento y el destino final del grano de cacao y sus derivados, de esta manera se generan muchas "percepciones" o ideas relacionadas con los demás actores, con una mejor posición o que ganan mucho más con su participación, o permite que algunos manejen mucha más información y tengan una mayor capacidad de negociación frente a los demás actores y ello generalmente despierta situaciones de desconfianza y poca voluntad de trabajo en equipo integrado. Así sucesivamente se definen los diferentes actores en la cadena de valor del cacao.

Aplicando el análisis del diamante competitivo de Porter, los factores responden a:

1. Se buscó rivales regionales o se diseñó la estrategia para la formación de rivales regionales fuertes, que en el caso de las ocho regiones cacaoteras son los agricultores de cada zona, para que haya una buena competencia dentro de las regiones.

2. Lo que se buscó fue determinar si hay una relación entre la dotación de recursos naturales y el desarrollo por cada región, y además si existe especialización de los recursos humanos en determinadas tecnologías.

3. Los clientes son cada vez más exigentes en el mercado.

4. Las agrupaciones de instituciones, empresas y organizaciones que contribuyen al desarrollo de un determinado sector cacaotero, logrando identificar la función de los mismos, compartiendo información, resolviendo los problemas de procesamiento y promoviendo ideas innovadoras.

Por lo que se definirá cada actor de la cadena de valor del cacao:

\section{Integrando el análisis de la cadena de valor y el diamante competitivo de Porter}

a) Los productores. Se concentran en diferentes regiones del país: San Martín, Junín, Cusco, Ucayali, Huánuco, Amazonas, Cajamarca y Piura, representando el 96 \% del total de la producción nacional (Romero, 2016).

La Tabla 1 muestra el crecimiento de la producción anual de cacao desde el año 2000 al 2016. En el año 2000 los departamentos con mayor producción de cacao fueron Cusco y Ayacucho con 8943 y 6297 toneladas métricas, respectivamente; los siguientes años desde 2001 al 2012 se han desarrollado pequeñas variaciones de incremento en producción de cacao para el departamento del Cusco y de 2001 al 2013 la producción de cacao para el departamento de Ayacucho se ha mantenido constante, sin embargo, en este mismo lugar los años desde 2014 al 2016 la producción de cacao se ha reducido. Por otro lado, los departamentos de San Martín y Junín son los que han incrementado la producción de cacao, principalmente, desde el año 2002 hasta el 2016 y actualmente estos dos departamentos son considerados como los de mayor producción de cacao para el Perú, siendo representado alrededor de más del 40 \% del total. Los departamentos con menores producciones de cacao de 2000 al 2016 son: Ucayali (393 a 8622 T), Huánuco (1968 a 6491 T), Amazonas (2922 a 4276 T) y Jaén (547 a 904 T). Existe la información de producción de cacao en grano en la categoría "otras regiones", las que pueden ser consideradas: Tumbes, Piura y otras menores. Por tanto, con datos del año 2016, las zonas de producción de cacao de mayor a menor son: San Martín, Junín, Cusco, Ucayali, Huánuco, Ayacucho, Amazonas y Jaén. El Perú ocupa el tercer lugar en el continente americano en producción 
de cacao, luego del Ecuador y Brasil, según información del año 2017 su participación es de 120 058 toneladas (INEI, 2018).

Se analiza esta situación del sector, considerando las herramientas prescritas, la que mejor se aplica es del diamante competitivo de Porter, pues es el resultado del trabajo coordinado del sector público (gobierno) con el gremio de productores de cacao y el aporte de la cooperación internacional, que permitió también marcar otro récord de 129842 hectáreas cultivadas de cacao en el 2017. Socialmente es favorable pues se generaron 9900000 de jornales, beneficiando de manera directa a 90000 familias, y de manera indirecta a 450000 personas, principalmente de la selva en el año de 2017.

Geográficamente los productores se ubican en zonas rurales, muchas veces en zonas agrestes, vírgenes y hasta en conflicto social narcoterrorista. Este último, es el caso de las áreas del valle de los ríos Apurímac, Ene y Marañon (VRAEM), por ejemplo, en Puerto Amargura (Ayacucho), el cultivo de la coca y el narcotráfico son actividades económicas de la población.

Tabla 1. Evolución de la producción anual de cacao en grano por regiones (En toneladas métricas)

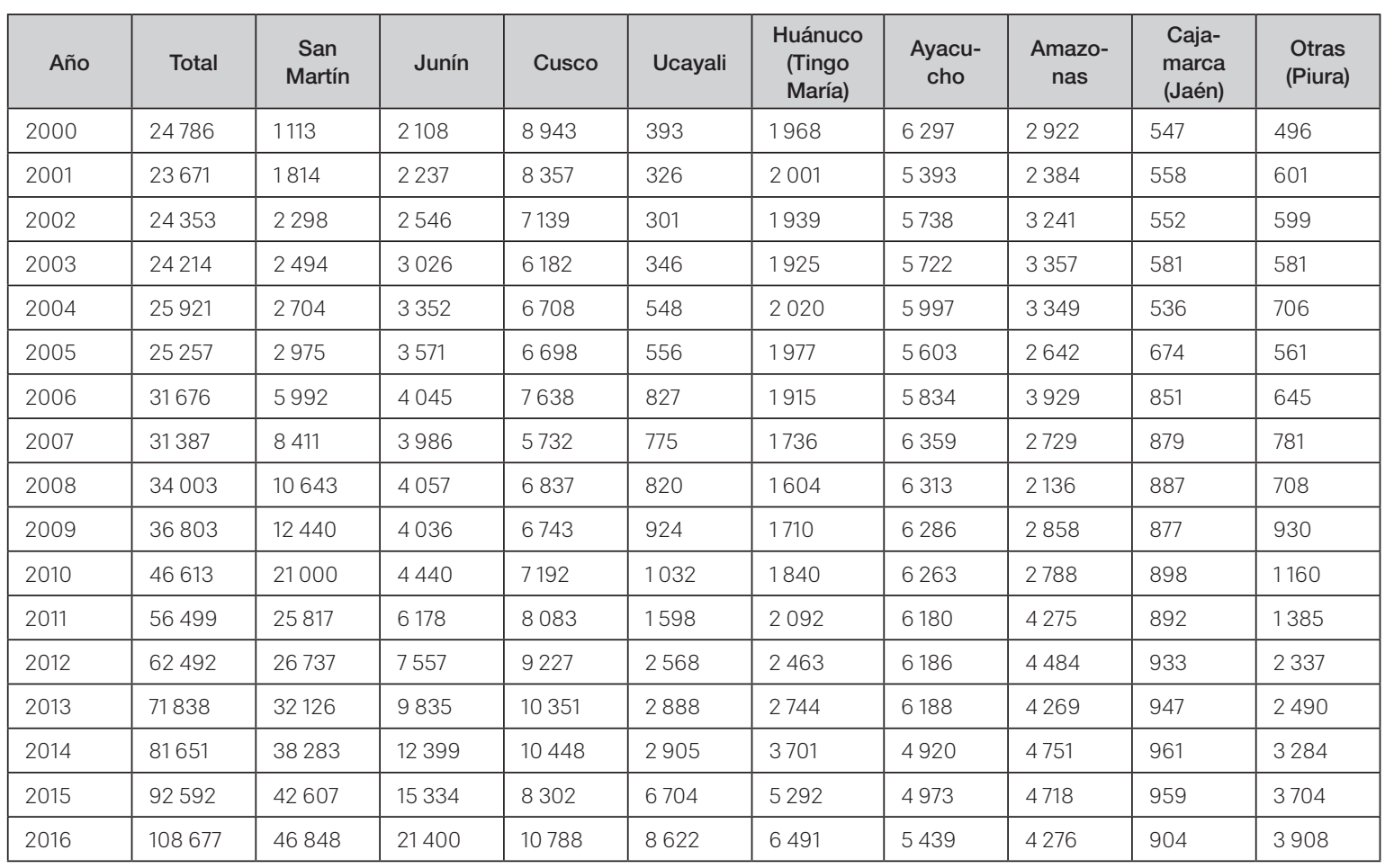

INEI (2018) y Romero (2016).

Con base en los estudios de investigación y consultorías de diversa índole realizadas en dichas zonas del país, existen elementos comunes que se pueden considerar genéricos en los productores cacaoteros, mostrando deficiencias gerenciales organizacionales. Sin embargo, se están se experimentan actualmente mejoras en la logística interna, lo que implica selección de terreno, substratos nutricionales, viveros, almácigos con pequeñas plantas de cacao provenientes de semillas mejoradas, siendo introducidas progresivamente y muestra de ello, son las experiencias en la región de San Martín, donde los agricultores están siendo organizados y man- 
tienen vínculos con organizaciones mejor posicionadas como la Cooperativa agraria cacaotera ACOPAGRO (Peñaloza, 2002).

Las operaciones que involucran actividades que promueven los factores de producción como preparación del terreno, instalación, manejo de viveros, trasplante, poda, control de plagas, abonamiento, cosecha y manejo poscosecha, también mantienen serias deficiencias, trayendo como consecuencias heterogeneidad en la calidad del grano del cacao.

Los agricultores o productores cacaoteros necesitan capacitación en temas técnicos sobre el manejo del suelo. El grano de cacao híbrido Colección Castro Naranjal \#51 CCN 51, es el que se ha introducido por sus virtudes de productividad y rendimiento industrial, además que sus plantaciones están siendo rehabilitadas y renovadas; superando las plagas, sobre todo, la persistencia e incidencia alta de moniliasis ocasionada por el hongo Moniliophtora roreri; la escoba de bruja ocasionada por el hongo Crinipellis perniciosa y la mazorca negra, ocasionada por el hongo Phytophthora palmivora. En la actualidad, se están manejando híbridos o varietales trinitarios, que proporcionan mayores rendimientos de productividad y niveles de grasa, tales como el ICS 1; ICS 6; ICS 39; ICS 60; ICS 95, (Selección del Colegio Imperial de Trinidad y Tobago) (García 2012). Asimismo, urge el aprendizaje de labores culturales como el manejo de la sombra permanente, introduciendo los cultivos frutales como acompañantes, además de las especies leguminosas y árboles maderables. Se requiere adecuado control cultural fitosanitario; y prácticas saludables de fertilización porque hoy en día buscan producir cacao orgánico para satisfacer la demanda del mercado internacional.

A todo esto se debe agregar la inocuidad del cacao: la calidad microbiológica, en cuanto a los hongos toxígenos; se concluye que aun siendo bajas las cantidades de ocratoxina A (OTA) halladas en los productos de cacao como en la formulación de chocolate en polvo y similares, y no representando una fuente primaria de OTA en la dieta, preocupa que dichos productos sean ampliamente consumidos por niños, quienes son más sensibles a los efectos de las micotoxinas (Chire, Valdivia y Ureña, 2014).

La logística externa, que trata básicamente del transporte del grano cosechado hacia la carretera o hacia las zonas de acopio (fermento y secado), tiene deficiencias, como es el caso de la región de Piura (Chulucanas y San Francisco y otros donde cultivan cacao blanco), observados de fuente primaria, desde las zonas de producción por parte del productor o del acopiador, con defectos propios desde el manejo del agricultor, vías de acceso precarias, medios de transporte, higiene y seguridad inadecuados. El mercadeo no está aún desarrollado a nivel de agricultores, carecen de técnicas y de poder de negociación, poca información de precios y las ventas se realiza al contado o a consignación.

A pesar de estas incipientes acciones, en las actividades de apoyo, se hacen denotados esfuerzos por mantener asistencia técnica en cuanto a: proveer insumos como yemas de cacao para el proceso productivo, capacitación, introducción de paquetes tecnológicos accesibles y viables. Socialmente, como el cacao corresponde a una zona rural, los hijos de agricultores tienen escolaridad limitada, con un acceso trunco a los sistemas educativos modernos de información y de tecnologías informáticas, consideradas hoy en día como decisivas en la educación inclusiva, y sin accesos a la seguridad social. Asimismo, en la parte individual "se visualizan como sujetos pasivos", con falta de poder y derechos.

Se están implementando estrategias clave para fortalecer la situación individual de los productores del sector cacaotero, como son las asociaciones y las cooperativas así como el mejoramiento de sus condiciones productivas que mejoren la competitividad en esta parte, 
pues son las formas jurídicas organizativas que posibilitan a las empresas agrarias competir en los mercados (Romero, 2009).

b) Los acopiadores. De la información recolectada in situ, los acopiadores locales en la región de San Martín, de las provincias de Moyobamba, Tarapoto (distritos de Chazuta y Saposoa), consideran al acopiador de bajos volúmenes menor a cinco toneladas y acopiadores de grandes volúmenes alrededor de 30 toneladas. Estos acopiadores son locales y los hay también a nivel regional. Algunos de estos actores acopian los granos de cacao y llevan a las cooperativas ofreciendo el grano de cacao según la calidad. Otros acopiadores a mediana escala (mayor a 5 toneladas y menor a 30 toneladas), concentran el grano de otros pequeños acopiadores y de los mismos productores con destino al mercado nacional como internacional, pero previamente realizan operaciones de limpieza y selección, como por ejemplo los acopiadores del grupo Romero (ROMEX), que son más organizados y tienen compromisos contractuales.

Actividades primarias: La logística del cacao fermentado y seco es precaria. El uso de costales de yute y de rafia para el pesado y transporte de cacao es de segundo o tercer uso; seguido por operaciones de selección y manejo del grano de cacao que requiere mayor secado. No conocen protocolos y normas peruanas para el manejo poscosecha del cacao, solo se negocia con el valor de humedad máxima de grano seco (7.5 \%) (INACAL, 2016). En el secado del grano de cacao, se logra el $8 \%$ de humedad, sin embargo, en la mayor parte de ellos se comercializa con humedad del 12 al 16\% en la región de San Martín (Centros de Producción Leoncio Prado y Juanjuí) (MINAGRI, 2003); poniendo en peligro biológico al grano como la contaminación por hongos y la producción de toxinas, como la OTA. Estos dos problemas, se deben a que los comerciantes intermediarios lo compran en estas condiciones, siendo ellos mismos, los que terminan dando el acabado del producto, por esta razón, incentivan las prácticas inadecuadas de poscosecha y obtienen ganancias a su favor ya que no secan el cacao como debe ser, haciendo que el peso del cacao sea mayor y por consiguiente facturando ingresos mayores a costa de una baja calidad del cacao (alta humedad). Según lo observado en las visitas in situ, esta situación es bien común en la región de San Martín (Tarapoto, Juanjuí, Uchiza y Nuevo Progreso), Junín (Satipo) y Piura (Chulucanas). La logística de salida concierne a la actividad de estibado desde la ruma, y el pesado en la balanza. El mercadeo consiste en la canalización del acopio de los granos de cacao hacia los acopiadores-comercializadores, 0 al industrial. En esta parte de la cadena, se busca la mejor negociación (integrativa) para ambas partes (productor acopiador), sin embargo, eso no se da, puesto que el acopiador posee mayor poder de negociación frente al agricultor por la calidad variable del grano de cacao.

Actividades de apoyo: La organización de los acopiadores es simple, cuentan con tres, cinco y hasta diez empleados en algunos casos; la infraestructura es variable pues hay quienes tienen locales grandes y otros tienen locales pequeños. No desarrollan muchas veces una planificación de sus actividades, actuando en función de los demandantes y del nivel de producción o de los acopiadores pequeños.

c) El acopiador-comercializador. Estos actores presentan limitaciones, las cuales destacan la capacidad productiva limitada. Sin embargo, se observa que el volumen de cacao que se produce es directamente proporcional al volumen de cacao que se comercializa dando una utilización de la materia prima del $100 \%$. Los granos son seleccionados por su calidad para luego ser almacenados.

La mayor parte de las asociaciones productoras cuenta con una capacidad limitada de asociación y agrupación, el recurso humano es poco capacitado para realizar una comercialización (con estrategia y de alcance), debido a falta de medios de información física o digital, no siendo así en las 
cooperativas organizadas como las que pertenecen a ACOPAGRO (Saavedra, 2007), que agrupa a más de 800 agricultores en Juanjuí (región de San Martín) o las que se encuentran agrupadas en la Asociación Peruana de Productores de Cacao (APPCACAO); que cuenta con 30000 productores de cacao, organizados en 25 asociaciones y cooperativas, ubicados en el norte, centro y sur del Perú.

La logística interna comprende actividades de valor vinculadas a la adquisición del grano cosechado por socios y no socios de las cooperativas y asociaciones a nivel nacional en cada región. Lo crítico aquí son las actividades de pesado, determinación de contenido de humedad y grado de fermentación del grano de cacao, con el objetivo de efectivizar castigos en el precio, por no cumplir los parámetros.

La logística de salida implica el transporte del grano acopiado ya sea en pequeños almacenes o de gran capacidad para embarcarlos a la capital de provincia o la capital del país. Los fletes pagados influyen negativamente sobre el precio en campo. Existe en la región de San Martín como Chazuta (Tarapoto) donde las condiciones de infraestructura vial terrestre han mejorado con respecto al año 2011 que fue visitado la primera vez. También en esta localidad se utiliza el río Huallaga como vía para transportar el cacao en balsas poco seguras sin considerar los tiempos de poscosecha del cacao.

Internacionalmente los precios del cacao en el mercado mundial están sujetos a fluctuaciones. Ghana, es el país, donde se determina el precio mínimo del cacao del mercado actual. El cacao de Ghana con certificación de Max Havelaar (la primera iniciativa de organismo de certificación de comercio justo en 1988) ha impuesto una bonificación, que se agrega al precio mínimo (CECU, 2007).

Figura 2. Precio promedio internacional CIF al contado del cacao en grano (Puertos de EE.UU.: y Europa) 2009-2017

\section{Precios $(\$ / T m)$}

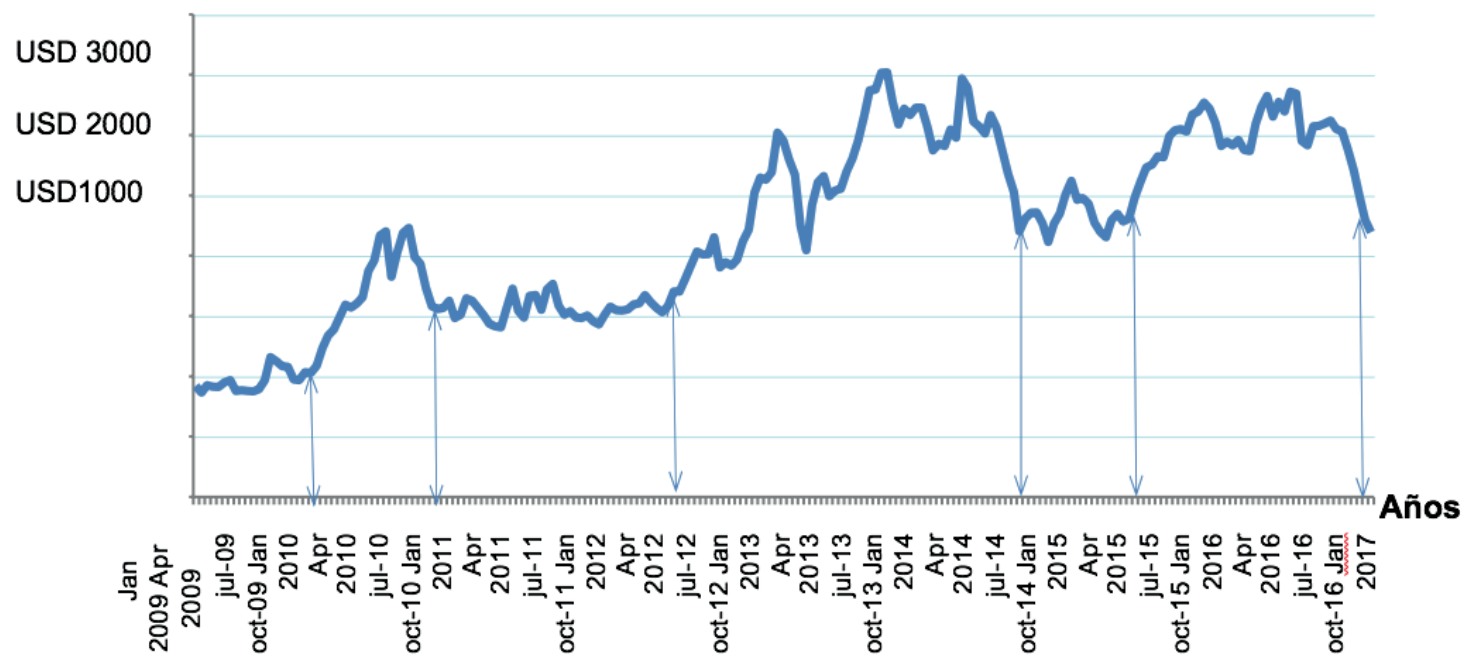

ICCO (2017)

Según la Figura 2, los precios internacionales del cacao muestran un comportamiento muy inestable, con grandes etapas de precios altos y precios bajos. A partir de noviembre de 2016 se estaría entrando a una etapa nueva de precios bajos. El precio del cacao en los merca- 
dos internacionales ha bajado desde USD 3000 a USD 2000 por tonelada, desde fines de 2016, debido a la sobreproducción mundial del grano de cacao, principalmente por la producción de África (Costa de Marfil), así como a las importantes existencias de cacao en los almacenes. A partir del año 2017, los precios se incrementaron en un 43 \% (alrededor de USD 2700 por tonelada) debido a que la sequía afectó los cultivos en el oeste de África, que da cuenta de más de dos tercios del abastecimiento global (ICCO, 2017).

La cadena del cacao en el Perú promete ser un negocio muy rentable. Sin embargo, los términos de intercambio del comercio internacional para los pequeños productores han caído, la diferencia existente entre los precios al productor y los precios pagados por el consumidor ha crecido enormemente y los productores de pequeña escala se están excluyendo de los mercados de alto valor añadido en ambos mundos, el industrializado y el denominado "en desarrollo". En estos momentos se está trabajando en el comercio justo y en la producción orgánica del cacao, así como en la prima de calidad (diferencial en el precio) en busca de conseguir un mejor precio.

Como lo señala Peñaloza (2002), el comportamiento del precio en campo muestra el alto poder de negociación de las empresas industrializadoras del grano de cacao, como ocurre en la región de San Martín.

A nivel nacional las regiones Amazonas (Bagua-Utcubamba), Cajamarca (Jaén-San Ignacio), Cusco (La Convención), Huánuco (Leoncio Prado), Junín (Satipo), San Martín (Saposoa, Juanjuí, Tarapoto, Tocache), Ucayali (Padre Abad) entre otras zonas del país, la distribución de los márgenes entre los agentes depende de sus capacidades de negociación, las cuales se posicionan mejor si tienen acceso a servicios de infraestructura. Los socios de las cooperativas determinan por medio de un proceso democrático cómo emplear la ganancia de su cosecha dentro de su comunidad.

La magnitud de los márgenes de comercialización a lo largo de la cadena de valor, afecta negativamente al proceso productivo y de distribución, por lo desorganizado de este eslabón.

En los estudios de Peñaloza (2002), se demuestra que en la región de San Martín el precio pagado al cacaotero está sujeto a la variación del precio promedio nacional pagado por las empresas procesadoras del grano, la calidad del grano producido, los fletes y también el tipo de cambio.

El consumo mundial de cacao sobrepasa las 3000000 de toneladas anuales. Los ocho mayores países productores de cacao son Costa de Marfil, Ghana, Indonesia, Nigeria, Brasil, Camerún, Ecuador y Malasia, que juntas representan el 90 \% de la producción mundial, 14000 000 de trabajadores están envueltos en su producción, 10000000 de los cuales se encuentran en África. Alrededor del 90 \% de la producción proviene de pequeñas propiedades, cuyos productores están entre los más pobres de su país; pues no cuentan con más de cinco hectáreas, poco organizados y con infraestructuras precarias que a menudo tienen que recurrir a intermediarios para vender sus cosechas. Se enfrentan a un mercado muy concentrado con pocos compradores, que venden el cacao en grano a una industria de transformación igualmente concentrada. De todo el cacao mundial, las dos terceras partes son procesadas en el mundo industrializado.

d) Los procesadores. Las actividades primarias comprenden a dos tipos de procesadores:

Procesadores primarios, en este eslabón se encuentran aquellos actores que se dedican a la fermentación y secado del cacao en grano y así venderlo a los procesadores secundarios. Las cooperativas de las regiones se encargan de acopio de cacao fresco, para fermentarlo y secarlo.

Procesadores secundarios, se caracterizan por ser industriales como micro empresas que abarcan el mercado nacional ofreciendo productos diferenciados con variadas presentaciones: 
cacao en pasta o licor de cacao, manteca de cacao y cacao en polvo como productos industriales y chocolates variados como producto terminado. Su cliente meta va desde una población con capacidad adquisitiva media hasta aquella que dispone de productos de alto valor agregado.

La tecnología del procesamiento existente en las plantas procesadoras es tradicional e intermedia. Hay zonas donde no existen tecnologías, como es el caso del VRAEM, donde se improvisa con el uso de materiales y equipos rústicos construidos en la zona.

La transformación artesanal está en manos de los propios productores socios y no socios de las cooperativas en la región de Ucayali (Cooperativa Campos Verdes), observado de fuentes primarias, en este caso conformada mayormente por mujeres. Estas organizaciones obtienen el cacao directamente de los productores, para después elaborar chocolates tipo taza y venderlo localmente en ferias, lugares turísticos, tiendas detallistas; también se dedican al procesamiento secundario y comercialización, ofreciendo barras de cacao y trufas.

Desde el año 2010 se está realizando un evento de carácter público-privado en conjunto con la cooperación internacional, "El Salón del Cacao y Chocolate," en donde los productores, fabricantes, emprendedores, exportadores de cacao y derivados se presentan con miras a elevar la calidad, productividad y oferta exportable del cacao peruano y posicionarlo en los mercados nacionales e internacionales, buscan integrar diferentes agentes económicos de la cadena productiva del cacao para gestionar conocimiento, intercambiar información, establecer redes de contacto y promover la actividad comercial del cacao y derivados en el mercado nacional e internacional.

Según MINAGRI (2003), en la región de San Martín (Juanjuí) tiene su sede la asociación ACOPAGRO, encargada de la comercialización del grano de cacao en mercados nacionales e internacionales, su radio de acción es el vale del Huallaga. La Industria Mayo con sede en la región de San Martín (Tarapoto), compra el grano de cacao de la zona y lo transforma en pasta de cacao, con una capacidad aproximada de 110 kg/hora. La Cooperativa Agraria "Naranjillo" con sede en la región Huánuco (Tingo María), transforma el grano de cacao en manteca y torta de cacao, con una capacidad aproximada de $500 \mathrm{~kg} /$ hora, compra grano de cacao mayormente de la región de San Martín y Ucayali. La empresa Cacao VRAE con sede en la región Ayacucho (San Francisco), vende cacao orgánico y convencional al mercado nacional e internacional, su acopio lo realiza en el VRAE. Se están haciendo esfuerzos en la gestión de este eslabón para que los actores se capaciten en aspectos de manejo, control e innovación.

e) Servicios de apoyo a la cadena. Contamos con servicio público y privado.

Público. El sector público provee en coordinación con agencias internacionales, a través del programa de Desarrollo Alternativo, liderado por el Estado Peruano, representado por la Comisión Nacional para el Desarrollo y Vida sin Drogas (DEVIDA), con el apoyo de la cooperación internacional de los EE. UU. y representada por la agencia de los EE. UU. para el Desarrollo Internacional (USAID), asistencia técnica para los productores de cacao a través de las escuelas de campo (Ríos, 2017). Los servicios de logística, financieros, administrativos y de certificación van por cuenta de los actores que trabajan en la cadena productiva en forma aislada.

En el año 2011 USAID firmó con la APPCACAO, un Convenio de Donación n. 527-C-0700002-CD354: "Buscando el Cacao de Oro", proyecto implementado de enero a julio de 2012.

Desde el año 2014, el gobierno, dentro del Plan estratégico nacional, a través de las mesas técnicas en cada región cacaotera, está desarrollando sus operaciones en las cinco líneas estratégicas de: asociatividad y organización; tecnología y calidad; agroindustria y valor; gestión empresarial y negocios; así como financiamiento, que hasta la fecha no ha tenido frutos 
contundentes, siendo reafirmada esta atingencia en la presentación del proyecto cacao seguro en julio de 2018, en donde uno de sus objetivos primordiales es tomar acción para mitigar y remediar el cadmio en los productos de cacao. La Dirección General de los Negocios Agrarios del MINAGRI, como aliado estratégico, articula y coordina las diferentes actividades de la cadena de valor del cacao. La Universidad de Hamburgo, Hamburg School of Food Science, Institute of Food Chemistry, la Corporation Sucursal de Perú Alianza Cacao Perú, la Cooperación Alemana al Desarrollo-Agencia de la GIZ en el Perú, y la APPCACAO, sostienen los diferentes proyectos de la agenda de la cadena de valor del cacao.

Las universidades nacionales y particulares han desarrollado trabajos de investigación sobre el cacao mediante las investigaciones y los proyectos presentados a través de INNOVATE PERÚ (Programa Nacional de Innovación para la Competitividad y Productividad del Ministerio de la Producción que fue creado el 24 de julio de 2014, mediante D. S. n. 003 2014) y PRODUCE (Unidad Ejecutora del Ministerio de la Producción, con autonomía económica, administrativa, financiera y técnica). Actualmente, la Universidad Nacional Agraria La Molina (UNALM) en Perú trabaja con la Universidad de Tolima en Colombia, el proyecto (Fondo Regional de Tecnología Agropecuaria) FONTAGRO titulado "Mejoramiento de la competitividad del sector cacaotero andino a través del desarrollo y la innovación tecnológica en la producción y transformación de productos de mayor valor agregado-innovación tecnológica cacao andino". También la UNALM, desde el año 2017 a la actualidad, está desarrollando el curso "Catadores en cacao y chocolate", que se encuentra en su tercera edición y en colaboración con el CBI (Centro para promover las importaciones de países en desarrollo) se ha llegado a un acuerdo para el desarrollo de un software especializado en evaluación sensorial de cacao y chocolate. Por su parte, el INACAL ha presentado públicamente el PNTP-CODEX CAC/RCP 72:2008 “Código de prácticas para prevenir y reducir la contaminación del cacao por ocratoxina A" y ha terminado de desarrollar el ENTP 107.303. CACAO Y CHOCLATE "Evaluación sensorial. Licor de Cacao", que definen importantes avances en la calidad del cacao peruano. Se puede tomar como referencia experiencias extranjeras, en el análisis de la cadena de valor del cacao en Colombia, plantea proyectos de investigación y transferencia de tecnología e innovación, que permitan recortar las brechas existentes en la situación actual (Contreras, 2017).

Sin embargo, a falta de una información amplia, holística, completa y útil, no se ha permitido aún la creación y concentración de una base de datos informáticos a nivel nacional.

Privado. Las diferentes instancias, organizaciones, empresas e individuos que cubren la totalidad de los servicios básicos necesarios para desarrollar la actividad cacaotera a un cierto nivel, corresponden al sector privado. Los servicios más demandados a lo largo de la cadena son el de certificación, asistencia técnica, logística, mejoramiento genético y mercadeo.

Las asociaciones productoras y las empresas de transformación secundaria continúan siendo el mayor cliente de prestadores de servicios privados. Para los productores en general, ya sean pequeños o medianos, el servicio de certificación es esencial para poder acceder a mercados internacionales que exigen una gama de especificaciones y requisitos que se centran en el modo de producción y traslado del producto. Estos se asocian con uso de agroquímicos e insumos en la producción, manejo de recursos naturales por parte del productor, procesos sanitarios y de inocuidad.

La asistencia técnica es fundamental para que la certificación se pueda realizar de manera exitosa, por lo que tanto las empresas que manejan la parte de producción como las que utilizan servicios de asistencia técnica y consultoría, generan ventaja competitiva dentro del 
mercado nacional e internacional. Los servicios de logística se contratan principalmente para exportación por parte de las empresas de transformación secundaria y comercialización (transporte, envío de muestras); ya que para el ámbito nacional es generalmente provisto por los actores inmediatos.

En general, todos los actores hacen uso en mayor o menor escala de servicios de transporte, certificación (orgánica o comercio justo), seguros, envíos de muestras, investigación de mercados.

El costo de estos servicios suele ser oneroso, sobre todo, para los productores pequeños de empresas de procesamiento secundario/comercialización que se localizan en lugares remotos y su capacidad adquisitiva es limitada. De ahí que sean los productores medianos y grandes, así como las empresas de transformación secundaria quienes solicitan dichos servicios con mayor frecuencia.

El eslabón de la cadena productiva que más apoyo tiene en la actualidad, en cuanto a servicios de asistencia técnica, es el de los productores. Se destacan entre estos servicios la generación y acceso a información, capacitación y la asesoría. Las debilidades de la cadena de valor del cacao están sujetas, principalmente, al volumen de producción comercializado, a la infraestructura disponible y a la débil gestión que realiza cada agente; tal como ocurre en la provincia de Los Ríos (Ecuador), los problemas en la comercialización del cacao, afectan directamente su rentabilidad y la economía de los pequeños productores, especialmente por la presencia del eslabón intermediario, en la cadena de comercialización (productor exportador), impidiendo que los beneficios lleguen al productor (Morales, 2013).

f) Las exportaciones. El 90 \% de la producción peruana del grano de cacao y sus preparaciones es destinado a la exportación. "La exportación peruana de cacao al mundo se expandió al 12,5\%, es decir, de 80000 toneladas el 2015 a 90000 toneladas en el 2016". Lo anteriormente citado ha sido corroborado por el cuarto boletín estadístico de la ICCO, cuando afirma que para el año 2016/2017, la proyección de la producción mundial del cacao provendrá de las Américas en un $17 \%$, cuando en el 2010, apenas llegaba al 9 \%; y el Perú es uno de los países que ha incrementado su producción y sus volúmenes de exportación.

La ICCO califica al Perú como el segundo productor mundial del cacao orgánico, albergando el $60 \%$ de los cultivares de cacao, poseedor de una increíble diversidad y variabilidad genética existente en el mundo, exportando el 36 \% del cacao que produce, además de ser una de las naciones con mayor calidad en la producción de cacao, la cual llegó a 56500 toneladas en el 2011 (Romero, 2016).

Las exportaciones de cacao en grano, entero o partido, a mayo 2012 alcanzaron los USD FOB 640000 (234 toneladas con precio promedio de USD FOB 2,73 por kilogramo) frente a mayo de 2011 con USD FOB 2590000 (a un precio de USD FOB 3,59 por kilogramo). En cinco meses, de enero a mayo de 2012, alcanzaron los USD FOB 6020000 (2309 toneladas).

En 1998, el Perú exportó 7931 toneladas en forma de manteca de cacao, cacao en polvo y chocolate, por un valor de USD 22300000 (MINAGRI, 2003). En los años 90, la empresa NEGUSA CORP. S. A., importaba cacao proveniente de Indonesia para la fabricación de pasta de cacao, manteca de cacao y chocolate en el Perú, y brindaba servicio para las empresas Costa y D'Onofrio. Concurrentemente, el área cultivada en el país aumentó de 17800 hectáreas en 1985 a 34786 hectáreas en 1995 (MINAGRI 2003). Las zonas productoras más importantes del país entre los años 1985-1995 se encontraban localizadas en las regiones de Cusco y Ayacucho que producían el 65,9 \% del total nacional, seguido por las regiones de Junín, Huánuco y Cajamarca con producciones del 27 \% del total. La producción nacional correspondió al 0,9 \% de los 2300 000 de toneladas de cacao producidas en el mundo en el 2011 (MINAGRI, 2003). 
Entre las principales empresas exportadoras en el año 2012 se encuentran: Amazonas Trading Perú SAC con el 59 \% del total exportado (149 toneladas), le siguen Natural Perú SAC con el 6 \% (5 toneladas), Machu Picchu Foods SAC con el $4 \%$ (4 toneladas), entre otros.

Las principales organizaciones de productores que exportaron cacao en grano en el año 2012 fueron: Asociación de productores Cacao VRAE con el 21 \% del total exportado (51 toneladas), y la Cooperativa Agraria Cafetalera "El Quinacho" con el $11 \%$ (24 toneladas). Esta cooperativa tiene tres certificaciones: Orgánica, Comercio Justo y UTZ. Una vez al año, representantes de las certificadoras llegan a Canayre a visitar los cultivos y asegurarse de que no usen plaguicidas. Los de Comercio Justo preguntan a los agricultores si les alcanza el dinero, si sus hijos estudian y si conocen cómo se manejan los recursos de la cooperativa.

Los principales destinos de las exportaciones de cacao en grano durante el año 2012 fueron: España con el 39 \% (USD FOB 248 874); Italia con el 21 \% (USD FOB 132 854); Holanda con el 20 \% (USD FOB 127 135); Bélgica con el 11 \% (USD FOB 67 941); EE. UU. con el 10 \% (USD FOB 63 949), respectivamente (MINAGRI, 2003).

En el año 2016, los principales destinos de este grano de cacao fueron principalmente Bélgica, Alemania, Suiza, Italia, Estados Unidos y Canadá. El Perú es el segundo país productor de cacao orgánico, después de República Dominicana. El 52,2 \% de la exportación fue de cacao en grano, de los cuales el 20 \% contó con la certificación orgánica y comercio justo (Romero, 2016).

Según Romero (2016), se exportó cacao en grano y sus derivados en 50000 toneladas, generando un valor de USD 118700 000, aproximadamente en el año 2015. Debido a que los consumidores europeos prefieren el cacao peruano, las ventas por exportaciones de cacao en grano sumaron USD 62800000 en el 2011, cifra que representa un aumento de 83,8 \%. Los despachos de cacao en grano aumentaron significativamente hacia Bélgica con una participación de 21,9 \% del total de los envíos, le siguen Colombia (21,6 \%), Italia (15,7\%) y Holanda (15,7 $\%)$. Asimismo, como segundo productor de cacao orgánico, orienta su producción a mercados de comercio justo.

Deficiencias en la cadena de valor. Del análisis realizado a los actores de la cadena de cacao por criterio estructural, se pudo identificar las siguientes deficiencias:

a. La cadena productiva del cacao está desarticulada.

b. La cadena está conformada por varios actores con capacidad productiva entre pequeña y mediana, que aún no satisfacen la demanda de los transformadores y comercializadores de producto terminado.

c. Desde la perspectiva gubernamental, la participación se está incrementando, pero solo en el aspecto productivo agronómico, desde el MINAGRI y pasando por ONG, hasta organizaciones extranjeras, con actividades que ha fomentado enseñanza en campo y apoyo técnico.

d. A nivel nacional, los procesadores industriales del cacao, grandes y pequeños, representan un número muy limitado, obteniendo solo productos industriales, dejando de lado productos de mayor valor agregado.

e. El problema sigue siendo los tipos de organización, la falta de diversificación de los productos, la calidad y volumen de producción como ventaja competitiva y los pocos productores con certificación orgánica.

f. El flujo de información a lo largo de la cadena es aletargado en los eslabones de producción y comercialización. Los actores en general reportan un flujo regular de información con sus clientes en cualquiera de los eslabones de la cadena. Las aso- 
ciaciones o empresas se encuentran localizadas en lugares con poco acceso a la información y medios de comunicación tecnológica. Esto limita en gran parte su acceso a información clave de cualquier tipo relacionada con su actividad económica. El flujo de información con sus proveedores en cambio difiere con la anterior, en cuanto a que esta se define como regular, siendo la razón principal de que la mayoría de los actores son de pequeña escala y por ende los proveedores de servicio cumplen con su obligación a un nivel básico.

g. El mayor número de actores se encuentra en el eslabón de producción, los cuales están agrupados en asociaciones (APPCACAO) las cuales abarcan casi en su totalidad a los productores de cacao en grano en el país. La productividad se ve afectada, principalmente, por la edad avanzada de los cacaotales, cultivares vulnerables a enfermedades, uso de insumos de baja calidad, limitada capacidad de gestión del conocimiento relacionado con prácticas de producción más eficientes y los contaminantes químicos (plomo y cadmio) y biológicos (OTA). Para el tema de producción, se le suman, exigencias del suelo y el clima, la toxicidad de los metales pesados, como el cadmio, pues es importante un monitoreo constante y encontrar formas de prevenir la contaminación en la cadena de producción del cacao y el tratamiento del fruto a lo largo del proceso productivo y prácticas culturales, en algunos casos.

h. El eslabón de transformación primaria presenta limitaciones de infraestructura por parte de los productores quienes no cuentan con instalaciones apropiadas para el fermentado y secado del cacao, lo cual determina en última instancia la obtención de requerimientos de calidad. También se relaciona con prácticas culturales y simplemente como un proceso que se ha realizado de cierta manera desde hace muchos años. Por esta razón, gran parte de la fermentación y secado del cacao de calidad se realiza por un reducido número de productores quienes tienen una mayor capacidad y los recursos necesarios para hacerlo, y son estos mismos, quienes proveen de la materia prima a aquellos que se dedican a la transformación secundaria.

i. La certificación también representa un reto tanto para el eslabón de producción como para el de transformación, tanto primaria como secundaria, sin embargo se vuelve más crítica para los productores (quienes realizan fermentado y secado), ya que en su mayoría no cuentan con los recursos necesarios e infraestructura para cumplir con los requisitos de acreditación.

j. Existen pocos procesadores secundarios porque los procesadores primarios cierran negocios con los exportadores de cacao en grano.

k. Otros factores identificados son la falta de recursos para crear capacidad instalada 0 ampliar la ya existente. Esta incluye maquinaria, herramientas y personal que maneje eficientemente los recursos. Para las pequeñas empresas es dificultoso encontrar proveedores fijos para los insumos, necesarios para el procesamiento del chocolate en sus diferentes presentaciones.

l. Los excedentes, la concentración del mercado en pocas manos y la presencia de la industria de transformación en los países europeos, forman parte de la raíz de los bajos precios pagados a las comunidades productoras, pero esto no es solamente consecuencia de los subsidios y la "competencia desleal" de los productos en el mercado mundial, sino que también juega un papel fundamental una compleja red de interrelaciones entre diversos factores. Entre ellos cabe destacar la liberalización del comer- 
cio y la competencia global que cada vez más se intensifica, la desregularización y el final de los acuerdos internacionales de las materias primas, las nuevas tecnologías y los costes de transporte reducidos, la deuda externa y la falta de alternativas $y$, por último, pero no menos importante, una concentración del poder del mercado en manos de poderosos compradores y en la industria transformadora, procesadora y de distribución que concentra el valor añadido en los países industrializados. Todo esto es combinado de manera que se pueda garantizar que en la mayoría de los mercados de materias primas el precio ya no regula la producción.

Propuestas de mejora en la gestión integral de la cadena de valor. Del estudio realizado se desprenden las siguientes propuestas:

a. Seguir fortaleciendo la cadena de valor del cacao, tal como lo asevera González (2014), cuando dice que el desarrollo de cadenas de valor tiene que centrarse en fortalecer las relaciones entre todos los actores que participan en la misma y asegurarse de que toda la información fluya desde los destinatarios finales del producto hasta los productores de base y viceversa, pues ayudará a los pequeños productores a identificar las oportunidades de mercado y mercadeo.

b. Dar valor agregado al cacao en la fabricación intermedia y artesanal de productos de consumo final (chocolates, trufas y chocolates variados), tal como concluye Barrientos (2015), en donde los países productores de cacao están haciendo esfuerzos por disminuir la exportación en grano, promoviendo que se dé valor agregado en las comunidades locales, dentro de las fronteras nacionales. Asimismo, es necesario implementar la estrategia de diferenciación, pues los demandantes internacionales están otorgando incentivos para la producción de granos diferenciados, lo que posibilita que los pequeños agricultores puedan aumentar la capacidad de comercialización frente a los compradores internacionales. En este caso, el cacao peruano tiene ventajas como: cacao fino o de aroma (CFOA) (Romero, 2016), cacao orgánico e innovación en productos de cacao, lo que debe ser aprovechado en beneficio de los pequeños agricultores. El futuro está en los mercados diferenciados.

c. Insistir en el apoyo a los actores desprotegidos de la cadena a nivel nacional (procesador primario, procesador secundario y comercialización), ya que se observa una concentración de recursos y asistencia por parte de actores tanto nacionales como internacionales para la mejora del cultivo de cacao en zonas mencionadas. Actualmente, varias organizaciones trabajan conjuntamente en aportación de recursos financieros, técnicos y humanos destinados a la renovación de cacaotales; testeo de cultivares resistentes a enfermedades, mayor rendimiento y calidad, capacitación y transferibilidad de mejores prácticas productivas con enfoque orgánico y sostenible.

d. El gobierno debería incrementar el apoyo con paquetes tecnológicos de transformación, desde el Ministerio de la Producción pasando por ONG, hasta organizaciones extranjeras. Incrementar las áreas de producción del cacao en las diferentes zonas del país. Ríos (2017) reafirma, cuando dice que el Perú, posee una política pública abierta y pragmática que no discrimina cultivares, fomenta el desarrollo de la asociatividad local y la inversión tanto pública como privada de manera diversificada. En particular, ha sabido aprovechar la intervención de la cooperación internacional con proyectos de carácter nacional, así como promover cacao para exportación como CFOA, sin im- 
portar el cultivar. El trabajo de varias cooperativas de productores están priorizando el cultivo de CFOA, desde los años 2000-2002.

e. Seguir apoyando iniciativas como la de García (2012); en donde publica la segunda edición de "Catálogo de Cultivares de Cacao del Perú", la que incluye 73 cultivares distribuidos en cuatro grupos genéticos: Trinitario (11), Forastero (7), Criollo (4), y Nacional (6). Además, se agregan a ellos, los cultivares Misceláneos (5), cultivares Huallaga (10), cultivares Ucayali-Urubamba (10), cultivares Marañón (9), y cultivares Nativos (2). Asimismo, se incluyen nueve árboles promisorios Selecciones Híbridas de la UNAS (SHU); que tiene como objetivos: (i) capacitar a curadores de bancos de germoplasma y jardines clonales, en la identificación y/o verificación de la identidad genética de los cultivares de cacao existentes en el país, y (ii) orientar a los funcionarios de agencias gubernamentales y no gubernamentales, empresarios privados y extensionistas, en la toma de decisiones bien informada sobre los atributos agroindustriales de estos cultivares validados y recomendados a nivel comercial por prestigiosas instituciones de investigación internacional, nacional, y de promoción y competitividad agraria.

f. Proponer modelos de organización en las diferentes zonas de producción de cacao como estrechar lazos con países consumidores de chocolate para transferir tecnología con convenios bilaterales (Guzmán, 2013).

g. El fortalecimiento del APPCACAO, como eje facilitador y arena concertadora de alianzas y colaboración entre los actores existentes de la cadena, así como aquellos prospectivos será de vital importancia para el desarrollo de los eslabones de transformación y comercialización.

h. Los procesadores primarios y secundarios se pueden beneficiar considerando que una gran parte de la producción nacional es orgánica, dando un claro enfoque al mercado orgánico como meta principal. Este tipo de mercado tiene actualmente una gran proyección de crecimiento en la próxima década. Es importante mencionar la importancia y reputación que tiene Perú como un país biodiverso.

i. Fomentar el consumo interno del cacao y chocolate, resaltando los beneficios para la salud por ser fuente antioxidante (Perea-Villamil, Cadena-Cala y Herrera-Ardila, 2009).

j. Es un reto revertir las importaciones del chocolate, considerando que nuestro país ofrece una diversificada oferta de cacao con el que se puede elaborar los más exquisitos chocolates, para el mercado interno y externo.

k. Proponer alianzas de carácter técnico: mejorar el manejo del cultivo, la calidad del producto, propiciar generación de información técnica y operativa e incrementar la capacidad empresarial de las organizaciones rurales, para posicionarse en el mercado internacional. Esto tiene como fin mejorar la calidad de vida de los productores y sus comunidades, ya que el cacao por ser un agronegocio rural, mejora los ingresos y calidad de vida de las comunidades donde se desarrolla (Ickis et al., 2009).

l. En cuanto a los centros especializados, urge la necesidad de crear laboratorios de cacao en planta, cacao en grano, cacao en pasta y en chocolate, así como laboratorios para controlar la calidad microbiológica, toxicológica, nutricional, funcional, fisicoquímica y sensorial.

m. INNOVATE PERU y PRODUCE deberían poner más atención a proyectos de investigación y transferencia de tecnología e innovación en el cacao, ya que en la revisión de la megabase Springer Link, se encontró que desde el 2000 al 2017, la generación de ar- 
tículos científicos sobre cacao ronda las 1461 publicaciones, de los cuales alrededor del $25 \%$ son artículos vinculados con ciencias agrícolas y solo un $7 \%$ de los mismos provienen de Latinoamérica; sin embargo, resulta relevante observar que más del $85 \%$ de los artículos e investigaciones sobre cacao en los países latinoamericanos están vinculados con agricultura (Arvelo et al., 2017).

n. El uso de prácticas agrícolas orgánicas y su certificación, utilizando plantas que absorban cadmio para lograr la inocuidad; así como evitar la incineración de residuo orgánico.

o. Proponer alianzas de largo plazo entre los actores diversos, para asistir en áreas como asesoría técnica, servicios de certificación, generación de información técnica y operativa para la consecución de proyectos cacaoteros.

p. Promover la creación de mercados sostenibles y de valor agregado de productos agrícolas de micro, pequeños y medianos empresarios rurales (MYPES), así como el apoyo a comunidades e instituciones locales comprometidas con el desarrollo sostenible.

q. Enfocar la producción a través de escuelas de campo con el fin de mejorar la producción de las zonas, con la formación de pequeños productores de cacao orgánico, en temáticas de la producción de los derivados del cacao (chocolates orgánicos); formación de catadores y técnicos de calidad en el manejo integral del cacao.

r. Buscar que las organizaciones se enfoquen en mercados donde prevalezca la certificación ambiental, la calidad de vida de los productores y la protección de la biodiversidad alrededor de las fincas cacaoteras.

s. Proponer estudios económicos a la cadena de valor del cacao.

De este análisis, el mercado del cacao peruano utiliza la "diferenciación" como estrategia de posicionamiento en el mercado mundial. Tal como señala Rueda et al. (2018), la demanda de cacao está experimentando un ritmo sin precedentes, al incrementar los mercados, que exigen una calidad más especializada. Para estos mercados, no solo los atributos objetivos del producto son importantes, sino también las descripciones de su origen, modo de producción y sostenibilidad ambiental, es decir la demanda de productos diferenciados.

El cacao orgánico como estrategia de posicionamiento. Según la Consultora SASE-KIPU (2007) el cacao orgánico se produce bajo condiciones ambientalmente sostenibles, por lo general en zonas de bosques húmedos y, a diferencia del cacao convencional, se cultiva en asociación con otras especies vegetales y con sistemas de conservación de los ecosistemas propios de cada región. Estos sistemas de producción permiten un mayor control de las enfermedades endémicas de los cultivos extensivos, pero generalmente tienen un menor rendimiento por hectárea. Los principales productos derivados del cacao orgánico son los granos de cacao, el licor o pasta de cacao, la manteca de cacao y el polvo de cacao o cocoa. Si bien la ICCO no cuenta con datos oficiales sobre el porcentaje de cacao orgánico producido, las estimaciones de los pocos estudios que se han realizado al respecto oscilan entre el 1 y el $8 \%$ de la producción mundial, y con una tendencia claramente creciente. El cacao peruano es del tipo aromático, caracterizado por su alto contenido de grasa (Chire, Orosco, Valdivia y Ureña, 2014), que le confiere un alto valor comercial en el mercado internacional y con un gran potencial para la producción de cacao orgánico como cultivo en sistemas agroforestales.

Además, existen varios países que cuentan con producción en proceso de conversión y certificación entre ellos Perú y otros países de América como Cuba, Brasil, Ecuador, Haití, Honduras, y Panamá. Es importante reconocer que los productores de cacao africanos parecen tener mayores oportunidades porque el cacao africano convencional es valorado como de mejor 
calidad, lo cual puede limitar el precio que se estaría dispuesto a pagar por el cacao orgánico de los países de América Latina. La producción orgánica debe ser certificada por una agencia autorizada en otorgar certificaciones internacionalmente, como por ejemplo SKAL (con sede central ubicada en Holanda, y cuyo sello es requerido para el cacao orgánico y sus derivados que se venden en Europa y Japón), OCIA (con sede central en Estados Unidos, y cuyo sello es requerido para el café orgánico que se vende en Estados Unidos y Canadá) u otras como BIOLATINA, IMO/ CONTROL, NATURLAND, BIOSUISSE, UTZ KAPEH.

Una de las ventajas más importantes de la certificación es la posibilidad de obtener un mejor precio por el producto. Así, si los granos de cacao convencional se venden según la cotización de las Bolsas de Londres y Nueva York (durante el 2005 el precio osciló alrededor de \$ 1 500 por toneladas), el cacao orgánico se vende a precio de Bolsa + USD 200, y el cacao orgánico y certificado FLO (Comercio Justo) se vende a USD 1600 + USD 350. En relación con este mercado de comercio justo, se estima que en los últimos años las transacciones en él vienen aumentando a una tasa promedio de $20 \%$ anual y que la tendencia es creciente. Con dicho mercado, el cacao se compra a un precio mayor y directamente a cooperativas de pequeños productores que cultivan sus tierras de manera ecológica. Los mayores beneficios son invertidos en proyectos comunitarios ligados a la salud, la educación o al medio ambiente.

\section{Conclusiones y recomendaciones}

Del estudio se desprenden las siguientes conclusiones:

Labores de manejo agronómico deficientes, la asistencia técnica requiere que sea más incisiva en el manejo del suelo, en la introducción de cultivares híbridos resistentes a las diferentes amenazas, con una fertilización y manejo integral de plagas responsables.

Se observa que la producción está dada en pequeñas extensiones, menos de tres hectáreas por lo que se propone implementar más formas asociativas que aseguren organizaciones eficaces y eficientes, permitiendo a los productores asegurar ofertas en el mercado interno y externo en diversas zonas del país.

La infraestructura, como apoyo de la cadena de valor del cacao, presenta altos costos de transporte interno en la mayoría de centros de producción, dado que los huertos están localizados lejos de los centros de acopio, utilizando como vías de comunicación trochas carrozables y en algunos casos solo se puede tener acceso a través de la vía fluvial, como se observa en el VRAE, San Alejandro y Chazuta en San Martín, por lo que se propone solicitar apoyo gubernamental.

La labor de poscosecha que corresponde a las operaciones de las actividades primarias de la cadena de valor son deficitarias. En el fermentado y el secado no se sigue recomendaciones de las normas técnicas peruanas, por lo tanto, se debe capacitar constantemente al cacaotero en buenas prácticas de poscosecha para obtener cacao en grano con contenido de humedad máximo de 7.5\%.

La producción actual del cacao está orientada a la venta de cacao en grano y transformación de productos industriales (commodities) por lo que se debe incentivar el desarrollo de empresas de elaboración de productos con valor agregado (Chocolates y pasta de cacao finos), negocios rentables de economía social y solidaria; para mercados nacionales e internacionales del comercio justo. 
No existen cadenas de comercialización que sean articuladas en la información y permitan mejoras para los actores de los eslabones, se propone interrelaciones entre las empresas o formación de clusters.

El precio del grano de cacao y sus derivados industriales está supeditado al precio de las bolsas de productos en el mundo, siendo bajo el margen asignado a los actores de la cadena, este depende de cada zona productora y de la calidad, por lo que se propone una estrategia de diferenciación como cacao fino o de aroma, cacao orgánico e innovación en productos de cacao.

Los actores directos como parte de la cadena de valor del cacao son: productor, acopiador, acopiador-comercializador, procesador (primario y secundario) y exportador. Los actores indirectos de la cadena de valor del cacao son los de servicios público y privado.

Se plantea la perspectiva de considerar al producto "cacao peruano", en el largo plazo, como generador de oferta sostenida, para una demanda creciente nacional y mundial, no solo como cacao orgánico y cacao fino o de aroma, sino también como "cacao funcional-fuente de energía", que incentiva e incrementa la producción y el procesamiento en diferentes productos con valor agregado.

\section{Referencias}

Arvelo, M. A., González, D., Delgado, T., Maroto, S., y Montoya, P. (2017). Estado actual sobre la producción, el comercio, y cultivo del cacao en américa. San José, C.R.: Instituto Interamericano de Cooperación para la Agricultura, Fundación Colegio de Posgraduados en Ciencias agrícolas-IICA.

Barrientos, P. (2015). "La cadena de valor del cacao en Perú y su oportunidad en el mercado mundial". Semestre Económico, 18 (37), 129-156.

CECU. (2007). "El sello de garantía de los productos de comercio justo". Guía publicada por la Confederación de consumidores y usuarios (CECU). Madrid. Programa subvencionado por el Ministerio de Sanidad y Consumo / Instituto Nacional del Consumo.

Contreras, C. (2017). "Análisis de la cadena de valor del cacao en Colombia: generación de estrategias tecnológicas en operaciones de cosecha y poscosecha, organizativas, de capacidad instalada y de mercado". (Tesis de Magíster en Ingeniería Agrícola). Universidad Nacional de Colombia.

Chire, G., Valdivia, R., y Ureña, O. (2014). "Ocratoxina A en cacao y derivados. Medidas Preventivas". Ciencia e Investigación 17(1), 9-15.

Chire, G., Orosco, P. J., Valdivia, R., y Ureña, O. (2014). "Determinación de las propiedades físicas, químicas y termo físicas de diferentes granos de cacao del Perú". En: Fito, P., Andrés, A. M., Arguelles, A. L., y Ortola, M. D. (Eds.). Actas CIBIA IX (3), 545-551.

García, L. (2012). "Catálogo de Cultivares de Cacao del Perú". Lima. Q \& P Impresores S.R.L. Dirección General de Competitividad Agraria. Dirección de Promoción de la Competitividad Dirección de Información Agraria. MINAGRI.

González, L. (2014). Análisis de la cadena de valor de la caoba en las cooperativas agroforestales del Valle de Sico-Paulaya e implementación del plan de negocio. (Tesis de maestría). Tegucigalpa-Honduras.

Guzman, J. H. (2013). "Competitividad de la quinua perlada para exportación: el caso de Puno". Ingeniería Industrial (31), 91-112.

Hernández, R., Fernández, C., y Baptista, M. P. (2010). Metodología de la Investigación, 5a. ed. CDMX, México: Mc Graw Hill.

ICCO. (2018). Organización Internacional del Cacao. https://www.icco.org/statistics/cocoa-prices/ monthly-averages. html?currency=usd\&startmonth=01\&startyear=2015\&endmonth=12\&end year $=2017 \&$ show=graph\&option=com_statistics $\&$ view $=$ statistics $\&$ ltemid $=114 \&$ mode $=$ custom \&type $=1$. 
Ickis, J., Leguizamón, F., Metzger, M., y Flores, J. (2009). "La agroindustria: campo fértil para los negocios inclusivos". Academia, Revista Latinoamericana de Administración (43), 107-124.

INACAL. (2016). "NTP 2451-2016: Grano de Cacao. Especificaciones". Instituto Nacional de la Calidad (INACAL)

INEI. (2018). Instituto Nacional de Estadística e Informática. webinei.inei.gob.pe:8080/SIRTOD1/inicio.html

MINAGRI. (2003). "Caracterización de las zonas productoras de cacao en el Perú y su competitividad". Informe final elaborado por el Programa para el Desarrollo de la Amazonía-PROAMAZONIA. Lima-Perú.

Morales, F. (2013). "Los productores de cacao tipo nacional en la provincia de los Ríos Ecuador: Un análisis socioeconómico". (Tesis para la obtención del grado de Magíster Scientiae en Extensión Rural). Universidad Nacional de Viçosa. Minas Gerais-Brasil.

Peñaloza, J. (2002). Competitividad de la cadena de valor del cacao en la región San Martín. (Tesis de grado de Economía). Universidad Nacional Agraria La Molina. Lima-Perú.

Perea-Villamil, J. A., Cadena-Cala, T., y Herrera-Ardila, J. (2009). "El cacao y sus productos como fuente de antioxidantes: Efecto del procesamiento". Revista de la Universidad Nacional de Santander (41), 128-134.

Rueda, X., Paz, A., Gibbs-Plessl, T., Leon, R., Moyano, B., y Lambin, EF. (2018). "Smallholders at a Crossroad: Intensify or Fall behind? Exploring Alternative Livelihood Strategies in a Globalized World". Business Strategy and the Environment Businees Strategy and the Environment (27), 215-229.

Porter, M. (1990). "La ventaja competitiva de las naciones". Harvard Review 85 (11), 69-95.

Ríos, F. (2017). Estrategias país para la oferta de cacaos especiales: Políticas e iniciativas privadas exitosas en el Perú, Ecuador, Colombia y República Dominicana. Bogotá, Colombia: Fundación Swisscontact Colombia.

Romero, C. (2016). Estudio de cacao en el Perú y el mundo. Situación actual y perspectivas en el mercado nacional e internacional al 2015. Lima, Perú: Ministerio de Agricultura y Riego-Dirección de Estudios Económicos e Información Agraria.

Romero, R. (2009). "Formas asociativas en la agricultura y las cooperativas". Estudios agrarios (41), 37-66.

Saavedra, J. (2007). Factores socio-económicos en adopción de tecnologías para el manejo Integrado del cultivo de cacao en la cooperativa ACOPAGRO, Huallaga Central-San Martín. (Tesis de Magíster Scientiae en Innovación Agraria para el Desarrollo Rural). Universidad Nacional Agraria La Molina. Lima-Perú.

SASE KIPU. (2007). “Plan operativo del cacao Región Amazonas”. Lima, Perú: Ministerio de Comercio Exterior y Turismo / SASE / KIPU. 